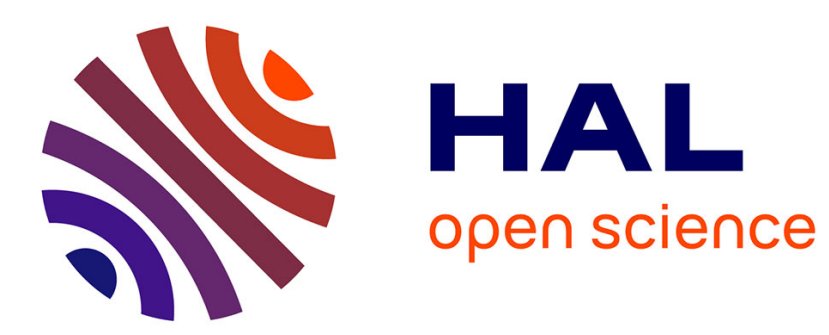

\title{
Le design de l'information : textualisation, documentarisation, auctorialisation
}

Manuel Zacklad

\section{To cite this version:}

Manuel Zacklad. Le design de l'information : textualisation, documentarisation, auctorialisation. Communication \& langages, 2019, 199, pp.37-64. 10.3917/comla1.199.0037 . hal-02934472

HAL Id: hal-02934472

https://hal.science/hal-02934472

Submitted on 30 May 2021

HAL is a multi-disciplinary open access archive for the deposit and dissemination of scientific research documents, whether they are published or not. The documents may come from teaching and research institutions in France or abroad, or from public or private research centers.
L'archive ouverte pluridisciplinaire HAL, est destinée au dépôt et à la diffusion de documents scientifiques de niveau recherche, publiés ou non, émanant des établissements d'enseignement et de recherche français ou étrangers, des laboratoires publics ou privés. 


\title{
Le design de l'information: textualisation, documentarisation, auctorialisation
}

\author{
Manuel Zacklad
}

Laboratoire Dicen-IdF, CNAM, Paris

\section{Introduction}

Les enjeux liés au design de l'information numérique sous toutes ses formes sont bien sûr de la première importance dans un contexte où les applications de l'internet et du web sont au cœur de la plupart des innovations économiques et sociales. Très souvent, malheureusement, la problématique du design de l'information est abordée uniquement sous l'angle de son traitement graphique et d'autres expressions, comme celle d'architecture de l'information, tendent à lui voler la vedette quand il s'agit d'aborder des dimensions plus structurantes. Pourtant, comme nous le rappelons dans un texte récent, le design n'est pas limité aux aspects graphiques ${ }^{1}$. Dans une acception francophone du terme de design, nous proposons d'abord de distinguer le design d'auteur du design systémique qui retiendra notre attention ici.

Le design systémique, qui correspond à l'activité de la très grande majorité des designers professionnels, recouvre l'ensemble des phases amonts du processus conception ce qui le différencie des phases aval de conception technique détaillée et de fabrication proprement dite. Cette conception amont implique de prendre en compte plusieurs dimensions des artefacts. En nous inscrivant dans la continuité de plusieurs traditions méthodologiques au sein du design ${ }^{2}$ nous distinguons différents niveaux de mise en œuvre correspondant, du plus concret au plus abstrait, à la forme, la fonction, l'expérience utilisateur ou usage et la valeur symbolique. Mais nous introduirons également un autre principe de description de l'artefact à concevoir, qui fait varier la perspective selon les différentes dimensions de la médiation qu'il opère, que nous approfondirons ici: l'information comme contenu ou comme texte, comme acte de langage

\footnotetext{
${ }^{1}$ Manuel Zacklad, «Design, conception, création : vers une théorie interdisciplinaire du Design ». Wikicreation, 2017. http://www.wikicreation.fr/fr/articles/934.

2 Par exemple, Quarante, Danielle. Eléments de design industriel. 3e éd. Paris: Economica, 2001.
} 
coproduisant les selfs de l'auteur et du récepteur et enfin comme support matériel de la gestualité ou d'inscriptions pérennes, constituant alors un document.

Nous commencerons par rappeler notre définition de l'information ancrée dans une vision anthropologique de la communication puis nous présenterons l'approche ternaire de l'information proposée par M. Buckland ${ }^{3}$ qui est en phase avec notre typologie. Dans un deuxième temps, nous reviendrons sur la notion de dispositif pour introduire les dispositifs d'information et de communication dont nous donnerons des exemples. Ceci nous permettra, dans une troisième partie, d'aborder le design de l'information consignée dans toute sa richesse et sa complexité, combinant les problématiques de la textualisation, de l'auctorialisation et de la documentarisation.

\section{L'information et la communication dans la sémiotique des transactions coopératives}

Notre vision de la communication est basée sur notre cadre d'analyse de l'action et de l'activité, la « sémiotique des transactions coopératives », dans lequel le terme de transaction est emprunté à Dewey et Bentley ${ }^{4}$. Notre référence à la sémiotique signifie que nous nous situons dans le courant des analyses sémiotiques de l'activité selon lequel la narration structure assez largement la cognition ${ }^{5}$. Elle ne signifie pas que nous ne nous intéressons qu'aux dimensions sémiotiques même si celles-ci retiennent naturellement notre attention.

Voici notre définition des transactions coopératives ${ }^{6}$ :

Une transaction coopérative est une mise en commun de ressources personnelles et le cas échéant collectives qui engagent des participants, réalisateurs et bénéficiaires, dans la réalisation d'une performance commune associée à la production et ou à l'acquisition d'un artefact porteur de valeur, une cuvre, et au développement de leur expérience personnelle (Fig. 1). La transaction se réalise par une succession de transformations permettant de passer des ressources à l'auvre. La performance est évaluée en tenant compte à la fois du résultat, l'cuvre, de l'expérience acquise par les sujets et de l'investissement qu'ils ont consenti. Le terme de «performance » renvoie à la description de l'action dans le schéma

\footnotetext{
${ }^{3}$ Buckland, Michael K. «Information as Thing ». Journal of the American Society for Information Science 42, no 5 (1 juin 1991): 351-60. https://doi.org/10.1002/(SICI)1097-4571(199106)42:5<351::AID-ASI5>3.0.CO;2-3.

4 Dewey, John, et Arthur, Fisher, Bentley. Knowing and the Known. Beacon Press, 1949.

${ }^{5}$ Bruner, Jerome Seymour. Car la culture donne forme à l'esprit: de la révolution cognitive à la psychologie culturelle. Traduit par Yves Bonin, Eshel, 1991.

${ }^{6}$ Voir aussi Zacklad, Manuel. «Le travail de management en tant qu'activité de cadrage et de recadrage du contexte des transactions coopératives ». Activités, vol. 10, $\mathrm{n}^{\mathrm{o}}$ 10-1, avril 2013. journals.openedition.org.proxybib.cnam.fr, doi: $10.4000 /$ activites. 650
} 
narratif de Greimas ${ }^{7}$, qui contient cinq temps : virtualisation, compétence, performance, évaluation, rétribution. Elle correspond à la réalisation "proprement dite de l'action rendue possible par la compétence positive $»^{8}$. Selon la part prise par chaque sujet dans la co-production, les transactions sont plus ou moins symétriques. Dans certaines transactions, l'effort est surtout consenti par un des transactant et la séparation entre réalisateur et bénéficiaire est tranchée. Dans le cas des transactions agonistiques, le résultat peut être bénéfique pour un participant mais négatif pour l'autre. Ce cadre s'applique à l'analyse des activités collectives mais également individuelles. Dans ce dernier cas, le même sujet est alternativement l'actant réalisateur et l'actant bénéficiaire qui réalise les évaluations intermédiaires et finales.

Le terme de communication est bien sûr un terme très polysémique. Sans parler de ses acceptions purement techniques dans le domaine des télécommunications il renvoie, notamment dans le champ des SIC, aux actions de communication mises en œuvre par des organisations pour s'adresser à un large public interne et externe et à la manière dont les médias, notamment les médias de masse, sont mis à contributions dans ces actions. Mais dans la droite ligne de notre approche pragmatiste, la communication est d'abord considérée dans le contexte de la communication interpersonnelle qui met en relation des sujets engagés dans la réalisation d'une performance commune, que celle-ci s'inscrive dans le cadre d'une conversation quotidienne, d'une réunion de travail, d'une négociation, d'une séance pédagogique, d'un débat scientifique ou d'échanges politiques.

Notre vision de la communication s'appuie sur les définitions de Dewey synthétisées par Quéré ${ }^{9}$ :

La communication correspond à des transactions interpersonnelles ò̀ les artefacts sémiotiques, notamment l'oralité et les textes, jouent un rôle central et dans lesquelles l'expérience a comme composante essentielle la capacité à adopter le point de vue d'autrui. Comme chez Dewey, le langage est considéré comme un " mode d'action » à part entière, le sens des mots et la comprébension de la signification « reposant sur un accord à la fois dans l'action et sur ses conséquences $\gg^{10}$. Cet accord sur la signification n'implique pas que les participants soient "d'accord entre eux» mais qu'ils se comprennent mutuellement quant au registre des actions possibles et de leurs conséquences. La performance de la communication tient simultanément à la qualité de l'expérience des

\footnotetext{
${ }^{7}$ Hébert, L. (s. d.). Algirdas Julien Greimas : Le schéma narratif canonique / Signo - Théories sémiotiques appliquées. Consulté 22 mai 2018, à l'adresse http://www.signosemio.com/greimas/schema-narratifcanonique.asp

8 Ibid.

9 Quéré, Louis. Le modèle esthétique de la communication de John Dewvey. Centre d'Etude des Mouvements Sociaux: OP19, 2014, http://cems.ehess.fr/docannexe/file/3168/porto2014_quere2.pdf.

${ }^{10}$ Quéré ibid.
} 
participants et à celle des productions sémiotiques, même quand cette communication est partiellement agonistique, comme dans certaines situations de négociation.

Les transactions communicationnelles sont donc médiatisées par des artefacts dont la fonction sémiotique est essentielle ou prime sur d'autres fonctions, artefacts sémiotiques qui sont notamment au cœur de notre définition de l'information. Parmi ces artefacts médiateurs nous introduirons une distinction principalement axée sur la différence entre la langue orale et la langue écrite. Alors que les supports gestuels de l'oralité ont un caractère éphémère, les supports de l'écrit doivent avoir un support pérenne. Mais avant d'approfondir ces typologies revenons sur nos définitions de l'information.

Dans la lignée de la tradition documentaire des sciences de l'information nous nous appuierons d'abord sur la vision ternaire proposée par M. Buckland dans son célèbre article "L'information en tant que chose »". Confronté à la diversité des sens du terme d'information, attestée par les définitions des dictionnaires, M. Buckland propose de distinguer trois principaux usages de la notion ${ }^{12}$ :

1. L'information comme processus, correspondant à l'acte d'informer ;

2. L'information comme connaissance, correspondant au savoir communiqué à propos d'un fait, d'un sujet ou d'un évènement ;

3. L'information comme chose, correspondant au fait d'attribuer à des objets tels que des données ou des documents une valeur informative.

\footnotetext{
11 Buckland, ibid.

12 Cette approche ternaire pourrait évoquer le vu, su, lu de R. T. Pédauque (Pédauque, Roger T. La redocumentarisation du monde. 1 vol. Toulouse: Cépaduès-éd, 2007) : support-vu, processus-su, connaissance-lu. Mais à la différence de celle de Pédauque, l'approche de Buckland, comme la nôtre, situe les enjeux documentaires dans la dimension du support de l'information, les dimensions du contenu (texte) et des actes de langage (autorité) caractérisant d'abord l'information avant de pouvoir être transférée par métonymie sur le document.
} 


\begin{tabular}{|l|l|l|l|}
\hline $\begin{array}{l}\text { Dimension de } \\
\text { l'information dans } \\
\text { notre perspective de } \\
\text { design }\end{array}$ & $\begin{array}{l}\text { Information } \\
\text { comme contenu }\end{array}$ & $\begin{array}{l}\text { Information } \\
\text { comme acte } \\
\text { langagier }\end{array}$ & $\begin{array}{l}\text { Information } \\
\text { comme support } \\
\text { (et inscriptions) }\end{array}$ \\
\hline $\begin{array}{l}\text { Usage du terme } \\
\text { d'information selon } \\
\text { Buckland }\end{array}$ & $\begin{array}{l}\text { Information } \\
\text { comme }\end{array}$ & $\begin{array}{l}\text { Information } \\
\text { comme processus } \\
\text { (action d'informer) }\end{array}$ & $\begin{array}{l}\text { Information } \\
\text { comme chose }\end{array}$ \\
\hline $\begin{array}{l}\text { Dans le contexte du } \\
\text { design de } \\
\text { l'information } \\
\text { consignée }\end{array}$ & Textualisation & Auctorialisation & Documentarisation \\
\hline $\begin{array}{l}\text { Équivalents possibles } \\
\text { pour l'information } \\
\text { orale }\end{array}$ & $\begin{array}{l}\text { Oralisation (logos } \\
\text { et pathos) }\end{array}$ & $\begin{array}{l}\text { Ethicisation } \\
\text { (construction de } \\
\text { l'éthos) }\end{array}$ & $\begin{array}{l}\text { Gestualisation, } \\
\text { ritualisation }\end{array}$ \\
\hline $\begin{array}{l}\text { Facette dominante } \\
\text { dans les SIC }\end{array}$ & $\begin{array}{l}\text { Les deux à la fois } \\
\text { information et } \\
\text { communication) }\end{array}$ & $\begin{array}{l}\text { Plutôt sciences de } \\
\text { la communication }\end{array}$ & $\begin{array}{l}\text { Plutôt sciences de } \\
\text { l'information }\end{array}$ \\
\hline $\begin{array}{l}\text { Description } \\
\text { conventionnelle des } \\
\text { dispositifs associés }\end{array}$ & $\begin{array}{l}\text { Information et } \\
\text { communication }\end{array}$ & $\begin{array}{l}\text { Dispositifs } \\
\text { communicationnel } \\
\text { s (présentiels) }\end{array}$ & $\begin{array}{l}\text { Dispositifs } \\
\text { informationnels ou } \\
\text { documentaires }\end{array}$ \\
\hline
\end{tabular}

Tab. 1. Les dimensions de l'information et ses déclinaisons dans différents contextes

Bien que M. Buckland soit d'abord un spécialiste de la bibliothéconomie et du document, cette vision ternaire peut s'appliquer aux situations de communication orales comme au situations de communication écrites relevant de l'information consignée. Selon notre perspective, focalisée sur les activités de design, il s'agit, comme nous l'avons dit en introduction, de concevoir l'information en prenant en compte les différentes dimensions de la médiation qu'elle opère, que nous approfondirons ici: l'information comme contenu, comme acte de langage coproduisant auteur et récepteur et enfin comme support matériel de l'oralité ou de l'écrit.

En prenant la perspective de l'information consignée, nous distinguerons dans l'information le point de vue du texte, qui met l'accent sur la signification dont elle permet la transmission, le point de vue de la construction de l'auteur dans la relation avec ses lecteurs et enfin le point de vue du support et de ses inscriptions, ou du document, constituant des traces matérielles plus ou moins dynamiques pouvant faire l'objet de différentes opérations logistiques au sens de Jeanneret ${ }^{13}$

\footnotetext{
${ }^{13}$ Jeanneret, Yves. « Logistiques de l'écrit ». Médium, no 10, 2007, p. 41-50. Cairn.info, doi:10.3917/mediu.010.0041.
} 
qui conditionne tant les relations sociales que la transmission des significations. La même analyse pourrait être faite en considérant l'information orale comme nous le proposons de manière hypothétique dans le tableau suivant.

Cette approche ternaire de l'information permet de positionner la théorie mathématique de l'information. Bien qu'utile dans le champ du traitement du signal celle-ci apparait comme étant très restreinte, à la fois parce qu'elle ne concerne que la perspective de la signification, sans prise en compte des enjeux liés aux actions interpersonnelles ni aux supports, mais aussi parce qu'elle restreint cette signification à une perspective probabiliste sans aborder les multiples facettes de la sémantique des langues fussent-elles artificielles. Restreindre le sens du terme d'information à cette théorie est contre-productif y compris dans le domaine de l'ingénierie des systèmes d'information où les questions des supports, des interfaces, de l'expressivité des modèles et des langages de programmation sont essentielles et bien éloignés, dans leur majorité, de la probabilité mathématique ${ }^{14}$.

La vision anthropologique qui définit l'information à partir de la communication entre des organismes vivant en donnant une place centrale à la communication humaine et qui souligne la diversité de ses acceptions, permet également d'identifier deux pôles au sein des sciences de l'information et de la communication. Un pôle qui privilégie l'acception de l'information dans ses dimensions sociales et interactionnelles, qui intéresse plutôt les sciences de la communication et un pôle qui privilégie la vision du support, des inscriptions et du document, du côté des sciences de l'information ${ }^{15}$. Les enjeux liés à la signification sont-eux partagés au sein deux courants : par exemple, les questions de sens ou de diffusion des représentations via les médias de masse sont fréquentes dans les sciences de la communication, celles liées à la sémantique et à la recherche documentaire sont très présentes dans les sciences de l'information.

Mais les chercheurs dans le domaine de la bibliothéconomie semblent s'être d'avantage appropriée le terme de sciences de l'information que les autres champs des SIC plus concernés par l'étude des relations interpersonnelles, de la communication des organisations ou enfin de l'étude des médias, pour ne prendre que quelques exemples. Même si, dans l'étude des média, l'utilisation du terme d'information pour désigner les nouvelles de presse brouille un peu les lignes. Une des raisons tient sans doute au fait que la bibliothéconomie a plus rapidement été confrontée à l'informatique et aux bases de données, des secteurs dans lesquels l'influence de la cybernétique avait largement popularisé le terme d'information

\footnotetext{
${ }^{14}$ C'est bien sûr à raison qu'Yves Jeanneret souligne également la différence entre le sens d'information dans cette théorie avec son sens en contexte social, Jeanneret, Yves. Y a-t-il (vraiment) des technologies de l'information? Édition revue et augmentée. Villeneuve d'Ascq, France : Presses Universitaires du Septentrion, 2007.

${ }^{15}$ Les inscriptions sont aussi des inscriptions dynamiques associées à la généralisation du numérique.
} 
dans son acception liée aux télécommunications, non sans susciter de nombreuses ambiguités. Les sciences de l'information sont donc principalement associées, au sein des SIC, aux enjeux de la gestion de l'information consignée. Dans la suite du texte, par convention, quand nous évoquerons des dispositifs informationnels nous ferons implicitement référence à l'information consignée tandis que pour évoquer des dispositifs donnant la priorité aux interactions synchrones, notamment en présentiel, nous parlerons de dispositif communicationnel.

Cependant, la généralisation des écritures numériques relevant des «documents pour l'action », qui rend possible une écriture collective fragmentaire et dialogale, dans les forums, les blogs, les wikis et les réseaux socio-numériques va singulièrement brouiller les frontières entre la sphère traditionnelle de l'écrit, caractérisée par une certaine stabilité et la sphère de l'oral, traditionnellement associée à l'interactivité et au dialogue. Auparavant, les progrès de l'audiovisuel avaient déjà rendus possible la diffusion à large échelle de la voix et de l'image sans toutefois permettre la participation de masse associée à l'internet et au web. Pour rendre compte de ces hybridations nous utiliserons le terme de dispositif infocommunicationnel correspondant à la rencontre inédite entre les pratiques de l'écrit et l'interactivité caractéristique des échanges en présentiel. Enfin, la catégorie des dispositifs transmédia, au sens où nous l'utilisons, qui permet de rendre compte de l'hybridation délibérée entre les dispositifs de communication qui articulent la programmation spatio-temporelle précise d'un évènement en présentiel et sa prolongation dans l'espace et le temps par un dispositif infocommunicationnel. Avant de donner quelques exemples de ces dispositifs, nous préciserons le sens que nous donnons à cette notion. 


\begin{tabular}{|l|l|l|}
\hline Type de dispositif & Modalité privilégiée & $\begin{array}{l}\text { Artefacts produit par la } \\
\text { transaction }\end{array}$ \\
\hline $\begin{array}{l}\text { Dispositif de } \\
\text { communication orale } \\
\text { interactif }\end{array}$ & $\begin{array}{l}\text { Interactivité, présentiel, } \\
\text { face à face, téléphone }\end{array}$ & Production verbale \\
\hline $\begin{array}{l}\text { Dispositif de } \\
\text { communication à } \\
\text { distance } \\
\text { unidirectionnel }\end{array}$ & $\begin{array}{l}\text { Diffusion de masse, } \\
\text { audiovisuel }\end{array}$ & $\begin{array}{l}\text { Production de contenu audio } \\
\text { et vidéo en mode non } \\
\text { interactif }\end{array}$ \\
\hline $\begin{array}{l}\text { Dispositif } \\
\text { informationnel } \\
\text { (informations } \\
\text { consignés) }\end{array}$ & $\begin{array}{l}\text { Écrits, échanges } \\
\text { asynchrones }\end{array}$ & $\begin{array}{l}\text { Production de textes (écrits, } \\
\text { film, musique) sur support } \\
\text { pérenne : documents }\end{array}$ \\
\hline $\begin{array}{l}\text { Dispositif info- } \\
\text { communicationnel }\end{array}$ & $\begin{array}{l}\text { Écriture interactive, } \\
\text { nouvelles brèves, } \\
\text { processus collaboratif } \\
\text { en ligne plus ou moins } \\
\text { synchrone }\end{array}$ & $\begin{array}{l}\text { Production de différents } \\
\text { contenus numériques, } \\
\text { souvent dialogiques et } \\
\text { fragmentaires : documents } \\
\text { pour l'action }\end{array}$ \\
\hline $\begin{array}{l}\text { Dispositif } \\
\text { transmédia } \\
\text { numérique }\end{array}$ & $\begin{array}{l}\text { Hybridation entre } \\
\text { évènementiel et info- } \\
\text { communication } \\
\text { numérique }\end{array}$ & $\begin{array}{l}\text { Production d'évènements } \\
\text { documentarisés }\end{array}$ \\
\hline
\end{tabular}

Tab. 2. Types de dispositifs d'information et de communication

\section{Transaction réseau et notion de dispositif transactionnel}

La plupart des transactions sont réparties dans l'espace et le temps. Que l'on pense à un projet de conception, à la rédaction d'un rapport d'étude, à un dossier rédigé à plusieurs par des étudiants, à la réalisation d'un tract par une association, aux pièces d'un procès en assises, à un dossier médical complexe, dans tous ces cas, les conversations et les écrits sont issus de multiples rencontres qui réunissent différents contributeurs selon un calendrier variable au sein d'espaces distincts. Cette prise en compte de la complexité de la distribution spatio-temporelles des actions est un des critères qui distingue l'analyse transactionnelle des analyses interactionnistes. Comme nous l'écrivions lors de notre analyse des transactions réseaux :

"L'analyse de l'action dans une perspective transactionnelle est rendue particulièrement difficile par le caractère fragmenté et réparti des procès transactionnels. En effet, non seulement la réalisation d'une transaction est-elle distribuée selon différents paramètres 
spatio-socio-temporels, mais une transaction donnée est susceptible d'en relancer d'autres d'une manière plus ou moins systématiquement dérivée de la première. Pour reprendre les métaphores géologiques de Deleuze et Guattari ${ }^{16}$, nous considèrerons que les transactions constituent des flux qui s'écoulent à des vitesses variables en s'entrecroisant à divers endroits. Toute situation d'activité effective peut ainsi être analysée en fonction de sa "généalogie ", qui l'inscrit à la fois dans un procès transactionnel direct et manifeste, mais également en fonction de procès transactionnels antérieurs auxquels les participants à la situation ne font pas nécessairement référence de manière explicite. ${ }^{17}$

Cette prise en compte de la complexité historique et spatiale des transactions explique notre recours au terme de dispositif. Comme l'a bien montré la sociologie des sciences et des techniques, dans le système de division du travail complexe qui est le nôtre, les artefacts médiateurs instrumentaux ou porteur de valeur sont le résultat de processus de conception et de fabrication très distribués, qu'il s'agisse de productions sémiotiques élaborées ou de machines, de véhicules, de systèmes de traitement de l'information qui s'appuient sur une fourniture de services en continu pour assurer leur maintien en fonctionnement : carburant, électricité, réseau internet... La plupart du temps, les artefacts médiateurs sont des "produits-services» impliquant de nombreux composants matériels (périphériques, logiciels, paramétrages...), documentaires (contrat, notice, système d'aide...), serviciels (connectivité, garantie, support, numéro d'appel...).

En suivant la manière dont P. Rabardel ${ }^{18}$ distingue les notions de «boite noire » et de «boite de verre » on peut considérer que selon les situations, les dispositifs d'information et de communication relèvent de tel ou tel régime de visibilité pour leurs utilisateurs. Dans un cours d'action routinier ou fluide, les composants sont agrégés et étroitement solidaires, ils sont transparents au sens où la boite noire invisibilise les composants. Si un imprévu survient ou si les acteurs transforment les caractéristiques de la situation, la complexité intrinsèque des artefacts doit être à nouveau prise en compte. La boite noire devient une boite de verre qui laisse voir la complexité des associations entre ses parties et qui implique une attention spécifique.

La même complexité est présente dans la prise en compte du milieu humain, car contrairement aux visions holistiques de la sociologie de la traduction ou de l'acteur réseau, il ne nous semble pas heuristique de traiter les sujets comme des nœuds de même nature que ceux qui constituent les artefacts techniques. Bien

\footnotetext{
${ }^{16}$ Deleuze, Gilles, et Félix Guattari. Mille plateaux. Éditions de Minuit, 1980.

17 Zacklad, Manuel. "Une théorisation communicationnelle et documentaire des TIC ». Socio-informatique et démocratie cognitive, édité par Claire Brossaud et Bernard Reber, Hermès science publications-Lavoisier, 2007, p. 20-35.

18 Rabardel, Pierre. Les hommes et les technologies; approche cognitive des instruments contemporains. Armand Colin, 1995. HAL Archives Owvertes, https://hal.archives-ouvertes.fr/hal-01017462.
} 
sûr, dans le déroulement d'une transaction, l'action entremêle les sujets et les artefacts. Bien sûr, il y a une codétermination entre les organismes vivants et le milieu technique et biologique. Mais les organismes vivants, les sujets humains au premier chef, contribuent à créer le milieu de manière délibérée en y introduisant des artefacts, en les associant entre eux, en définissant des mécanismes de liaison et des interfaces qui finiront par constituer des boites noires. Ce milieu est très largement le résultat de processus de conception intentionnels qu'il importe de saisir autrement que par le constat ex-post des associations entre tel contributeur et tel artefact.

Les selfs qui constituent le milieu humain sont individuels mais également collectifs car les sujets humains ont cette capacité remarquable de passer en « wemode », pour reprendre l'expression de G. Gilbert ${ }^{19}$, d'alterner entre un mode-je et un mode-nous. Qui plus est, ce mode « nous » est à échelle variable, le périmètre des acteurs se reconfigure en fonction des appartenances multiples qui sont les leurs, appartenances qui font souvent l'objet de négociations intenses.

Par ailleurs, les agencements collectifs sont eux-mêmes le résultat de formes contractuelles et conventionnelles très sophistiquées notamment dans le cadre des entreprises qui sont des espaces de création de rôles, de statuts, de modalités de coordination et de coopération médiatisées d'une infinie variété. Rappelons notre proposition de typologie des agencements collectifs correspondant à différentes pratiques : agencement familial, réseau relationnel, communautaire, organisationnel, marchand, territorial, éphémère... Ces agencements sont des construits culturels plus ou moins délibérés et normés qui conditionnent tant le mode-nous que le mode-je au sein du collectif.

Le dispositif correspond à l'ensemble des artefacts qui médiatisent l'activité des sujets au sein des agencement collectifs où se négocient les contours des selfs et où sont créés les artefacts porteurs de valeur. Ces transactions peuvent être simple ou réseau, plus ou moins distribuées dans l'espace et le temps, unique ou récurrentes. Quand ces artefacts ont une dimension sémiotique prédominante, en particulier langagière, les dispositifs sont des dispositifs d'information et de communication. Les dispositifs sont toujours, pour nous, des dispositifs de médiation ce qui nous rapproche des chercheurs dans le domaine de l'apprentissage qui parlent notamment de «dispositifs de communication et de formation médiatisés $»^{20}$.

19 Gilbert M. (2003), Marcher ensemble, Paris, PUF, Cité par Quéré, Louis. cems: OP32 / Nature et formes de l'émotion collective. http://cems.ehess.fr/index.php?3708. Consulté le 13 juin 2018.

20 Peraya, Daniel. «Médiation et médiatisation : Le campus virtuel». Hermès, no 25, 1999, p. 153. Crossref, doi: $10.4267 / 2042 / 14983$. 
Le sens du terme de «médiation » est donc en partie différent de celui qu'il a dans le domaine de la médiation patrimoniale comme chez Jeanneret et Rondot ${ }^{21}$ qui définissent les dispositifs de médiation comme étant spécifiquement destinés à véhiculer des contenus de médiation dans les institutions muséales. Notre concept de médiation est basé sur la psychologie socioculturelle de Vygotsky ${ }^{22}$, mobilisée dans la théorie de l'activité. Chez cet auteur, la pensée se développe grâce à l'intériorisation des symboles sociaux, notamment langagiers, qui deviennent des instruments psychologiques médiatisant le rapport du sujet à l'environnement et aux autres sujets. Se situant dans cette tradition, Peraya établit une distinction entre la médiatisation de l'activité des sujets qui est opérée par les artefacts et la médiation opérée par un sujet au bénéfice d'autres sujets. Bien qu'elle soit certainement pertinente dans le contexte de l'enseignement à distance nous ne la reprenons pas à notre compte à ce stade.

La médiation entre les selfs opérée par les dispositifs au bénéfice des activités individuelles ou des agencements collectifs s'appuie sur quatre composantes interdépendantes :

- un ou des espaces définis par leur architecture et des règles d'habitation dans lesquels se déroulent les transactions où se répartissent les sujets et artefacts ;

- une ou des temporalités et des protocoles associés incluant une durée et un rythme correspondant à la récurrence des interactions, prévoyant des temps d'activité collective et d'activité solitaire et permettant la rencontre entre les sujets et entre les sujets et les artefacts ;

- d'autres $\operatorname{artefacts}^{23}$ capacitants, matériels et sémiotiques ${ }^{24}$, situés dans l'espace et le temps et pouvant être, par exemple, du mobilier, des machines de traitement de l'information, de production d'énergie, de déplacement, d'enregistrement, des textes, des images, des paroles produites par les sujets, etc. structurant les mises en relation en définissant différentes formes de mise en visibilité ou de mise en écoute ;

- le cas échéant, l'état courant de l'œuvre en cours de réalisation ;

- les activités d'autres sujets nécessaires au fonctionnement des espaces et des artefacts mais ne faisant pas partie de la performance principale.

\footnotetext{
${ }^{21}$ Jeanneret, Yves, et Camille Rondot. « Médiation de la médiation au musée du Louvre. Des logiques de recherche au sein d'un projet politique, Abstract, Resumen ». Les Enjeux de l'information et de la communication, no 14/1 (2013): 131-47.

${ }^{2}$ Vygotski, Lev Semenovitch. Pensée et langage. Ed. Sociales, 1992.

${ }^{23}$ Nous parlons ici "d'autres artefacts" car l'espace et le temps peuvent aussi être considérés comme des artefacts médiateurs.

${ }^{24}$ Les artefacts sémiotiques on bien sûr aussi un support matériel. Les artefacts sémiotiques sont ceux dans lesquels la fonction sémiotique prédomine sur d'autres fonctions. Les fonctions matérielles sont moins sujettes à interprétation que les fonctions sémiotiques, elles s'objectivent plus facilement en particulier grâce à la réalisation de mesures.
} 
Dans cette définition, les sujets dont l'activité est médiatisée par le dispositif n'en font donc pas partie intrinsèquement. Le dispositif est pour partie au service des intentions convergentes ou conflictuelles des sujets et symétriquement le dispositif conditionne ou «modélise » l'activité des sujets. Les sujets ne peuvent pas agir à titre individuel ou collectif sans la médiation d'un dispositif et leurs actions, tout en visant une performance indépendante du dispositif de médiation instrumental, la réalisation de l'œuvre, contribuent à le faire évoluer de manière plus ou moins intentionnelle à travers le processus d'appropriation.

De ce point de vue, la conception d'un dispositif est toujours au service de la réalisation d'une performance et prescrit un cadre notamment informationnel et communicationnel permettant de l'atteindre, sachant que l'information et la communication peuvent être également l'objet de la performance. Mais, sauf dans les cas extrêmes où l'action est très standardisée et le milieu très stable, cette prescription est une prescription faible ${ }^{25}$ qui laisse une place importante à l'initiative des sujets dans le déroulement des transactions.

En s'appropriant le dispositif, les sujets le transforment de manière originale en fonction des circonstances. Ce faisant, ils se transforment eux-mêmes, ils font évoluer leur self en mode je et en mode nous, en modifiant leurs représentations et leur pouvoir d'agir. L'action vise donc selon nous trois finalités conjointes correspondant à trois types de « rationalité »: la création de nouveaux artefacts répondant à des besoins ou des désirs (rationalité substantive), la transformation des relations entre les selfs d'un point de vue individuel et collectif (rationalité agentive) et la transformation des dispositifs permettant l'action et les apprentissages (rationalité procédurale).

\section{Illustration des dispositifs d'information et de communication}

En suivant la distinction conventionnelle, mais non fondée théoriquement, entre un usage du terme d'information qui s'applique de manière préférentielle à l'information consignée et un usage du terme de communication qui correspond plutôt à l'oralité (tableau 1), nous proposerons ici des exemples des principaux types de dispositifs d'information et de communication permettant de montrer la diversité de leurs formats et leur hybridation. Ces typologies montrent comment le design de l'information numérique au sens d'information consignée est lui-

25 Hatchuel, Armand. "Coopération et conception collective - Variété et crise des rapports de conception ». Coopération et conception, édité par Gilbert de Terssac et Erhard Friedberg, Octarès, 1996. 
même imbriqué avec d'autres formes d'expression qui sont souvent complémentaires.

\subsection{Exemple de dispositifs de communication interactifs}

Les dispositifs de communication interactifs privilégient, comme leur nom l'indique, l'interactivité, le présentiel et le face-à-face. Ils correspondent aux discours, conversations, échanges en réunion qui exploitent la gestualité dont l'oralité est une composante. Chacun de ces dispositifs implique un espace, une temporalité et un agencement d'artefacts sémiotiques et matériels. Citons, par exemple les formes d'expressions suivantes :

- la conversation, le bavardage, la dispute ;

- la discussion approfondie, la négociation ;

- l'entretien formel ou ritualisé (évaluation, vente...);

- la réunion de travail, la séance de créativité ;

- le débat, le jury, la revue de projet ;

- la conférence (avec questions), le show....

Comme tous les dispositifs d'information et de communication, il se déclinent différemment selon le type d'agencement collectif auquel ils sont destinés. La réunion n'est pas de même nature dans un contexte familial, communautaire, de projet en entreprise, politique, etc. Pour un certain nombre de ces dispositifs, ils sont fréquemment complétés par des écrits et relèvent de la problématique du transmédia que nous examinerons plus bas. Les télécommunications et la diffusion quasi universelle du téléphone ont introduit une première révolution en permettant des conversations synchrones à distance. Elle se prolonge avec la numérisation généralisée qui offre des possibilités d'audio et de visio-conférence de plus en plus sophistiquées.

\subsection{Les dispositifs de communication audiovisuels}

Les dispositifs de communication audiovisuels étaient en nombre assez restreint du point de vue des supports techniques de diffusion avant la généralisation du numérique. Ils relèvent de formats d'expression extrêmement diversifiés qui se renouvellent sans cesse. Après le cinéma, la radio comme la télévision ont inventé de très nombreux types d'émissions qui se sont diffusés internationalement en créant des genres structurants, pour partie inspirés de la communication interactive en présentiel : entretien, conversation, débat, show... Les rôles entre les participants y sont bien sur très dissymétriques, avec une place importante jouée par le «star system» du côté des sujets réalisateurs des transactions, les présentateurs et autres animateurs. Les agencements collectifs sont territoriaux 
pour l'audiovisuel public et marchansd pour le privé, communautaires dans certains cas, avec des interpénétrations nombreuses. La numérisation des contenus a radicalement changé la donne avec la généralisation du replay et autres offres. Le flux est devenu stock rejoignant en partie les enjeux des dispositifs informationnels numériques.

\subsection{Les dispositifs informationnels}

Les dispositifs informationnels classique exploitent des supports pérennes pour diffuser les contenus et constituent des documents au sens que nous allons approfondir plus bas. Dans une approche dispositive, l'affiche, l'article ou le compte-rendu ne désignent pas uniquement le support du texte mais incluent l'ensemble des artefacts et le réseau d'acteurs qui permettent de les distribuer. Citons, par exemple, dans le domaine de l'écrit, les documents suivants :

- les affiches ;

- les monographies pour le grand public;

- les monographies spécialisées professionnelles ou scientifiques ;

- les journaux et les articles grand-public ;

- les journaux et les articles spécialisés dont scientifiques ;

- les lettres, les formulaires ;

- les notes de travail, les supports d'écriture temporaires ;

- les comptes rendus, les rapports ;

- les procès-verbaux, les textes officiels ;

- les supports de présentation;

- etc.

A ces documents relevant de l'écrit il faudrait rajouter ceux relevant d'autres formats d'expressions, les disques, et les fichiers audio pour le son, les photos et les vidéos pour l'image sur différents supports analogiques et numériques.

A nouveau, le type d'agencement collectif auxquels les formats d'expression sont destinés influence fortement la nature des dispositifs. La lettre n'a pas le même statut en contexte familial et amical qu'en contexte professionnel ou marchand. La numérisation généralisée a transformé les dispositifs informationnels selon différents axes en permettant d'une part, d'accéder aux contenus quasiment en tous lieux à travers le réseau internet quand ils sont « dématérialisés » et d'autre part d'introduire de nouveaux régimes d'interactivité qui étaient jusqu'alors réservés aux dispositifs de communication interactifs. 


\subsection{Les dispositifs infocommunicationnels numériques}

Les dispositifs infocommunicationnels numériques sont caractéristiques de la généralisation de l'internet et du web qui prolongent la numérisation des supports $^{26}$. Ils bousculent de manière profonde l'ensemble des dispositifs de communication et d'information précédents sans bien sûr les remplacer. Leur caractéristique majeure est de s'appuyer sur le numérique pour proposer une forme d'interactivité dialogique en mode texte qui peut être la finalité principale du dispositif ou qui vient enrichir la mise à disposition des contenus traditionnels mis à disposition en format numérique en permettant des commentaires relatifs aux cuvres. C'est la raison pour laquelle ils sont intrinsèquement infocommunicationnels. Nous avons proposé une typologie approfondie des dispositifs de médiation numérique dans un chapitre d'ouvrage consacré aux genres documentaires ${ }^{27}$ dans lequel nous présentions la typologie suivante, très légèrement remaniée ici ${ }^{28}$. Ces différents dispositifs peuvent se retrouver à l'état « pur» mais sont le plus souvent associés dans des formes hybrides:

- Dispositif de publication en mode pull : diffusion de documents numériques en mode pull via des portails documentaires, des bibliothèques de ressources multimédia pouvant être visionnées à la demande et ouverts aux commentaires et aux avis.

- Dispositif de diffusion et de publication en mode push : correspondance par messagerie électronique mais aussi circulation de contenus numériques qui sont «poussés » vers les utilisateurs via la messagerie, des SMS, des applications mobiles, en suivant, le cas échéant, des workflows plus ou moins automatisés.

- Dispositif rédactionnel : rédaction coopérative asynchrone ou synchrone d'un document par un groupe restreint de contributeurs allant de de l'usage $\mathrm{du}$ mode annotation des suites bureautique aux environnements en ligne de type « GoogleDoc » ou «Office 365 » en passant par les wikis ou des forges (p.e. sourceforge).

- Dispositif contributif en mode dialogal : ils sont caractéristiques des usages du web 2.0 et ils mettent plus l'accent sur l'interactivité que sur la production de contenus structurés, contrairement aux précédents, en

\footnotetext{
${ }^{26}$ Le terme de dispositif d'information et de communication ou dispositif infocommunicationnel qui correspond, par exemple, au nom que notre équipe de recherche avait utilisé pour nommer le laboratoire en 2009 « Dispositifs d'information et communication à l'ère numérique » est aussi utilisé par certains auteurs comme Gardiès, dans un contexte notionnel différent pour représenter «les liens entre la communication et l'information ainsi que les processus de médiation, ce qui se traduit par la dénomination intégrative de dispositif informationnel et dispositif communicationnel » Gardies, Cécile. Dispositifs info-communicationnels de médiation des savoirs: cadre d'analyse pour l'information-documentation. Université de Toulouse 2 Le Mirail, octobre 2012. HAL Archives Ouvertes, https://hal.archives-ouvertes.fr/tel-01725359.

27 Manuel Zacklad ; "Genre de dispositifs de médiation numérique ». Les genres de documents dans les organisations, Analyse théorique et pratique, édité par Louise Gagnon-Arguin et al., PUQ, 2015, p. 145-83

${ }^{28} \mathrm{La}$ liste contenait également les dispositifs transmédia que nous présentons dans la partie suivante.
} 
visant des communautés d'utilisateurs éventuellement très étendues, e.g. forum de discussion, plateformes de blog, plateformes de partage, etc.

- Dispositif contributif en mode plateforme : à la différence du dispositif précédent la plateforme n'a pas pour objectif principal la communication interpersonnelle mais vise à permettre la mise en relation des participants pour la production de services dans une logique d'économie collaborative, de sciences participatives ou d'évaluation.

- Dispositif attentionnel en mode flux : ils correspondent aux réseaux socionumériques (à l'heure actuelle Facebook, Twitter, Instagram, Pinterest, Google Plus, LinkedIn, etc.) et ils relèvent d'une intensification des dispositifs contributifs. Leur spécificité est leur position quasi monopolistique pour les plus grandes d'entre elles et leur fonctionnement inspiré du principe des téléscripteurs de presse actualisant en permanence un fil d'information pour capter l'attention des utilisateurs.

Les dispositifs infocommunicationnels dépendent également du type d'agencement collectif. Par exemple, un média social (dispositif contributif en mode flux) organise différemment la médiation au sein du grand public, de l'entreprise ou d'une communauté dédiée à l'atteinte d'objectifs partagés.

\subsection{Les dispositifs transmédia numériques}

Les dispositifs transmédia hybrides numériques font partie des dispositifs du

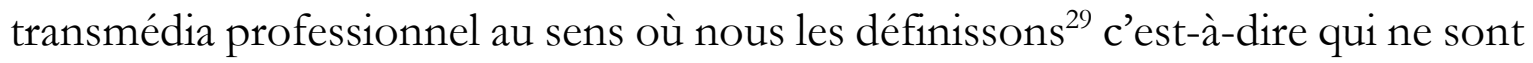
pas restreint à l'industrie des loisirs. Nous avions établi une distinction entre un transmédia (1) documentaire, lien entre plusieurs documents relevant de contextes médiatiques distincts, (2) ancré, lien entre un document et d'autre composantes spatio-temporelles de l'environnement-support (localisation par GPS, IoT, réalité augmentée...) et (3) hybride, lien entre toutes les composantes de l'environnement support intégrant, dans les cas les plus exigeants, la programmation temporelle, par exemple, dans la gestion d'un évènement. Sauf précision, quand nous faisons référence aux dispositifs transmédia numériques il s'agit du transmédia hybride.

Si l'on considère les transactions réseau avec une certaine profondeur temporelle, la plupart des dispositifs d'information et de communication sont, en fait, intrinsèquement transmédia. Ils le sont, par exemple, quand ils associent un dispositif communicationnel, une réunion et un dispositif informationnel, le compte rendu documentarisé de cette réunion diffusé à d'autres acteurs qui n'en faisaient pas initialement partie, puis archivé au sein d'une organisation. Ils le sont

${ }^{29}$ Manuel Zacklad « Organisation et architecture des connaissances dans un contexte de transmédia documentaire : les enjeux de la pervasivité ». Études de communication. Langages, information, médiations, nº 39, décembre 2012, p. 41-63. 
quand une consultation médicale est précédée et prolongée par un texte dans le dossier patient ou quand, dans la salle de classe, la pédagogie associe comme c'est le cas depuis fort longtemps, interactions en présentiel, manuels scolaires, cahiers des écoliers.

Mais dans ce dernier cas, par exemple, l'association entre les dispositifs communicationnels et informationnels fait l'objet d'une gestion ad hoc par l'enseignant qui met en place une forme de méta-dispositif articulant plusieurs dispositifs d'information et de communication qui, au moins pour les dispositifs informationnels, n'ont pas été spécifiquement conçu pour cette articulation : lien entre le cahier et le manuel, lien entre le manuel et les interactions en présentiel, lien entre le cahier, le manuel et les travaux des élèves, etc. La mise en œuvre du méta-dispositif repose en permanence sur les consignes de l'enseignant et la bonne coopération avec les élèves et les parents pour rester cohérent.

Par contraste, les dispositifs transmédia sont délibérément conçus pour permettre l'articulation dynamique entre, d'une part, des dispositifs communicationnels reposant sur la rencontre en présentiel et l'environnement support que constitue l'architecture des espaces et la programmation temporelle et d'autre part, sur différents dispositifs infocommunicationnel mis à disposition avant pendant et après la rencontre. Prenant la suite de l'acception large du terme de transmédia hybride ouvert aux dispositifs évènementiels que nous avons introduit en $2012^{30}$ Louise Merzeau ${ }^{31}$ en fournit une excellente illustration et en enrichit la conceptualisation dans l'analyse du dispositif mis en place à l'occasion des Entretiens du Nouveau Monde Industriel organisés par l'IRI et différents partenaires, dans ce qu'elle décrit comme «l'éditorialisation d'un évènement ». Elle montre comment ce dispositif articule un avant, un pendant et un après en combinant espaces physique et numérique.

Le succès des approches transmédiatiques est donc de permettre de décrire la manière dont des flux d'activité sont pris en charge par différents dispositifs d'information et de communication complémentaire. Cette description est bien adaptée à l'analyse et à la conception des activités de service impliquant des applications numériques qui sont centrales pour de nombreuses innovations actuelles. Elle est plébiscitée dans le design de l'expérience utilisateur qui vise précisément à intégrer de manière harmonieuse les différentes interactions du bénéficiaire avec les points de contacts du dispositif serviciel, interactions correspondant aux étapes de la co-production du service.

30 Zacklad ibid.

31 Merzeau ibid. 


\section{Les trois moteurs du design d'information dans une perspective dispositive : textualisation, auctorialisation, documentarisation}

Nous sommes maintenant en mesure d'aborder les enjeux du design d'information dans toute leur richesse et leur complexité, sans se limiter aux aspects graphiques, sachant que notre cible englobe l'information augmentée par l'interactivité des dispositifs info-communicationnels comme l'intégration de ces dispositifs dans des configurations transmédia. Comme nous l'avons signalé en introduction, dans cet article, nous insistons surtout sur à la nécessité de faire varier les points de vue sur l'information (contenu de l'information, support d'information, acte d'informer) pour mieux appréhender la dimension dispositive, plutôt que sur la problématique des niveaux de mise en œuvre (forme, fonction, usage, symbole) qui pourraient prolonger ce travail. Par ailleurs, nous visons ici l'information consignée, mais l'information orale, les dispositifs de communication, pourraient faire l'objet d'une réflexion similaire. La figure 1 schématise les différents points de vue qui seront développés. 


\section{Activités de design d'information ou de design de dispositifs informationnels ou infocommunicationnels}

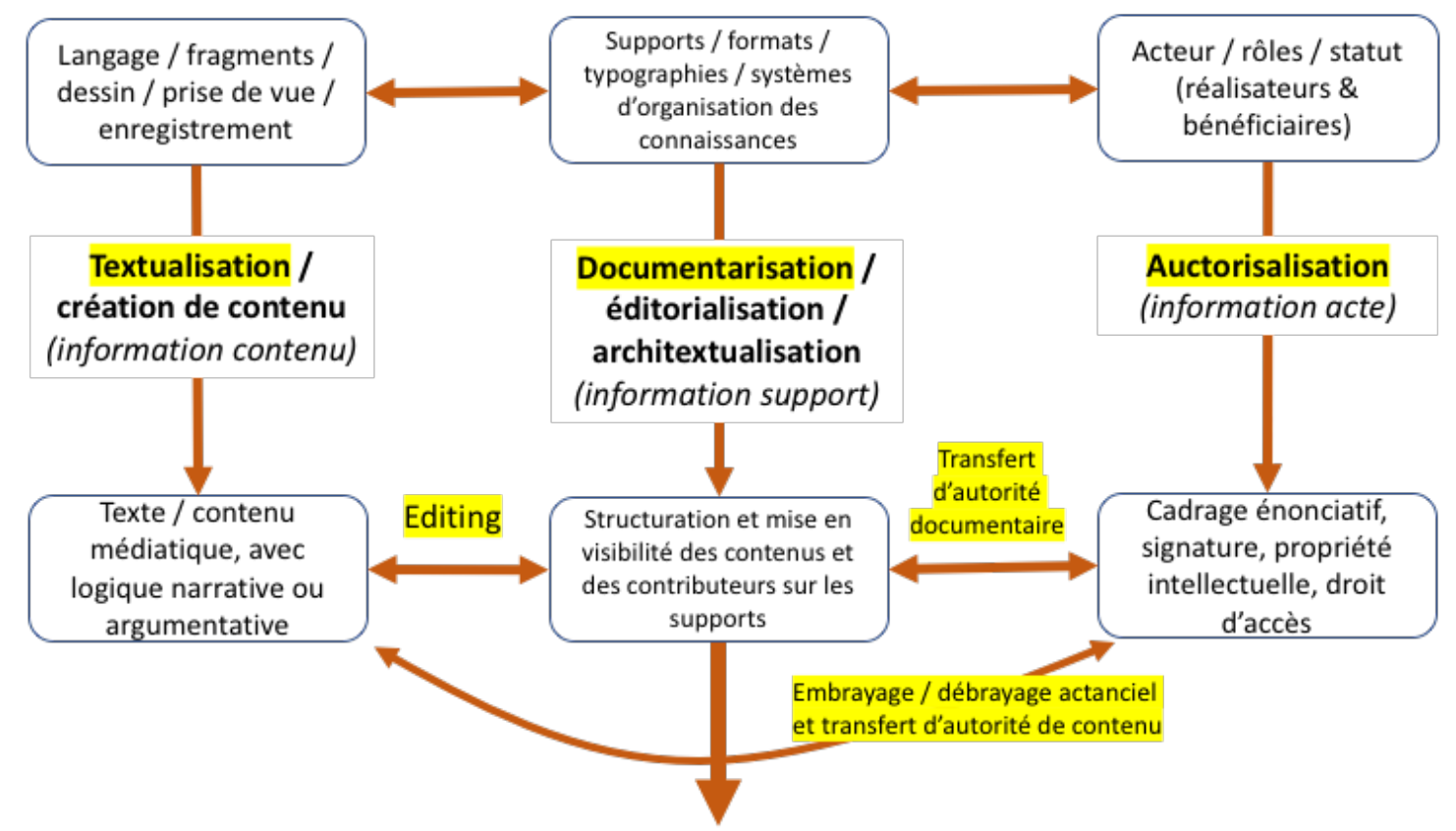

Information documentarisée, document autonome ou document réseau intégré au dispositif infocommunicationnel numérique

Fig. 1. Les trois moteurs du design d'information

\section{La textualisation ou le contenu informationnel}

La textualisation correspond à l'écriture ou plus largement à la production de contenu destiné à être consignée sur des supports pérennes. Le texte restitue une expérience pour en susciter de nouvelles. La notion de texte ou de contenu est envisagée de manière très large : il peut s'agir d'écrits de différentes natures, de schémas, de dessins, d'images, de vidéo, de musique et toute combinaison de ces formes d'expression.

La textualisation inclut toutes les opérations associées à la production de ces contenus. Très souvent elle s'appuie sur des contenus existants qu'elle réassemble de la manière originale, contenus eux-mêmes issus d'un travail de recherche d'information ou de documentation. Chaque forme d'expression ou langage dispose d'une syntaxe, d'une sémantique et de règles de style associées. Leur prise en compte relève largement d'autres spécialités que celles des sciences de l'information et de la communication, allant des études littéraires aux études cinématographiques en passant par les sciences du langage et nous ne chercherons pas ici à les décrire. Ce que nous prendrons en compte ce sont les interactions des processus de production de contenu avec les deux autres processus essentiels au 
design de l'information, l'auctorialisation et la documentarisation, qui permet l'ancrage sur le support.

\section{L'auctorialisation ou l'acte d'informer : construction des selfs à travers le texte et le document}

Dans toute transaction coopérative il y a une double construction des selfs et des artefacts médiateurs et parmi ceux-ci de l'artefact médiateur objet de la performance que nous appelons l'œuvre et des artefacts médiateurs capacitant correspondant au dispositif. La réitération de ces transactions contribue à la construction progressive du self qui s'appuie sur diverses prises de rôles. Le self tire notamment sa créativité de son engagement dans une multiplicité de situations transactionnelles auxquelles il participe, situations relevant de divers agencements collectifs. Cette construction du soi à travers les interactions verbales a été décrite par de nombreux auteurs. E. Goffman ${ }^{32}$ parle de construction de l'identité réalisée à travers les interactions quotidiennes. Lave et Wenger ${ }^{33}$ soulignent l'importance de la participation légitime périphérique pour l'intégration dans les communautés de pratique, participation qui se fait essentiellement à l'occasion d'interactions verbales.

Dans les transactions distribuées médiatisées par de l'information consignée, les mêmes processus sont en jeu. L'auctorialisation désigne pour nous le processus par lequel les identités se construisent à travers les textes et leurs supports documentaires : auctorialisation textuelle, le devenir auteur par le texte, auctorialisation documentaire, le devenir auteur par la documentarisation. Parmi les manifestations de l'identité, l'autorité du producteur d'information est souvent mise en avant. L'auctorialisation sera donc souvent abordée comme un transfert d'autorité issue du rôle du producteur d'information dans un agencement collectif au texte au document. Les transferts d'autorité textuel se manifestent par le biais de citations ou par l'adoption d'une structure énonciative ou d'un style associé à des rôles. L'auteur est un porte-voix qui agence les voix de différentes instances pour assoir la sienne. Dans l'auctorialisation documentaire, l'autorité s'exprime à travers le choix des supports, des genres éditoriaux, par l'utilisation de la signature d'une institution ou de son logo etc. (cf. Fig. 1).

\footnotetext{
32 Goffman, Erving. La mise en scène de la vie quotidienne 1 : La présentation de soi. Editions de Minuit. Paris: Les Editions de Minuit, 1973.

${ }^{33}$ Lave, Jean, et Etienne Wenger. Situated Learning: Legitimate Peripheral Participation. 1st edition. Cambridge England ; New York: Cambridge University Press, 1991.
} 


\subsection{Auctorialisation textuelle : embrayage et débrayage actanciel}

La production du texte implique une modélisation des positions actantielles du réalisateur qui se positionne par rapport à ses lecteurs en tant que membre légitime d'un collectif ou comme sujet indépendant assumant de manière pleine et entière sa subjectivité. Il peut s'exprimer à la première personne, comme narrateur ou en gommant tout trace de subjectivité, en s'appuyant notamment sur des systèmes d'organisation des connaissances standardisées comme dans les formulaires administratifs. Pour décrire cette relation d'influence réciproque entre la textualisation et l'auctorialisation nous utilisons les notions d'embrayage et de débrayage actantiel introduite par Greimas et Courtes ${ }^{34}$.

Une des manières d'aborder ces notions, par ailleurs assez complexes, est de s'appuyer sur l'interprétation fournie par Denis Bertrand ${ }^{35}$ cité par Fiorin ${ }^{36}$. L'embrayage est défini de la manière suivante :

"Elle [cette opération] "consiste alors pour le sujet de la parole à énoncer les catégories déictiques qui le désignent, le "je 》, l' iici» et le «maintenant 》: leur fonction est de manifester et de recouvrir le "lieu imaginaire de l'énonciation» par le moyen des simulacres de présence que sont je, ici et maintenant. Ces catégories se définissent par leur relation et leur opposition aux catégories débrayées. "17

Symétriquement le débrayage est :

" la possibilité de poser des ils, des alors et des ailleurs, c'est-à-dire de quitter l'inhérence à soimême et de se représenter des sujets et des choses sans rapport avec la situation de parole, comme dans une projection objectivante 》 (pp. 58-59). ${ }^{38}$

Cardon et Delaunay-Téterel ${ }^{39}$ montrent bien, dans le cas des blogs, comment « dans le monde de l'autoproduction amateur, la séparation de la personne et de son œuvre constitue toujours une étape cruciale et difficile à négocier ». Les blogs sont d'ailleurs analysés par ces auteurs comme des techniques relationnelles de production de soi.

\footnotetext{
${ }^{34}$ Courtés, Joseph, et Algirdas Julien Greimas. Sémiotique. Dictionnaire raisonné de la théorie du langage. Paris: Hachette, 1993.

35 Bertrand, Denis. Précis de sémiotique littéraire. Paris: Nathan Université, 2000.

36 Fiorin, Jose Luiz. «À propos des concepts de débrayage et d'embrayage : les rapports entre la sémiotique et la linguistique ». AS - Actes Sémiotiques, 15 février 2016. http://epublications.unilim.fr/revues/as/5605.

${ }^{37}$ Ibid.

38 Ibid.

39 Cardon, Dominique, et Hélène Delaunay-Téterel. "La production de soi comme technique relationnelle ». Réseaux no 138, no 4 (1 décembre 2006): 15-71. https://doi.org/10.3917/res.138.0015.
} 


\subsection{Auctorialisation documentaire}

Parallèlement à ces interactions entre auctorialisation et textualisation de nombreux enjeux sont liés aux choix des environnements-supports au sein des dispositifs. Par exemple, plusieurs auteurs se sont intéressés à la manière dont le livre papier, considéré comme le dispositif réseau emblématique de l'écriture créative jusqu'à la fin du $20 \mathrm{eme}$ siècle, est concurrencé par les nouveaux dispositifs éditoriaux de l'ère numérique comme le soulignent Neeman et al. ou Candel et Gkouskou-Giannakou ${ }^{40}$. En suivant ces auteurs, on peut considérer que chaque dispositif informationnel est porteur de formes d'auctorialité spécifiques. Si ces auteurs ne s'intéressent guère aux écrits administratifs et d'entreprise, il est possible de généraliser et de considérer que chaque dispositif informationnel inscrit les producteurs de contenu comme des auteurs légitimes dans un agencement collectif donné, que celui-ci soit communautaire, organisationnel, marchand ou territorial.

Le contexte actuel, caractérisée par la généralisation des documents pour l'action numériques ${ }^{41}$, donne lieu à de nouvelles formes d'auctorialisation. Parmi celles-ci, Broudoux ${ }^{42}$ en identifie une qu'elle nomme l'autoritativité et qu'elle définit comme une "attitude consistant à produire et à rendre public des textes, à s'autoéditer ou à publier sur le $W W W$, sans passer par l'assentiment d'institutions de référence référées à l'ordre imprimé». Toujours selon Broudoux, les nouveaux dispositifs de médiation numérique permettent de mettre en évidence "l'autorité informationnelle» composée de quatre types d'autorité combinées : l'autorité énonciative associée au nom et à la réputation de l'auteur, l'autorité de contenu définie par le genre éditorial, l'autorité du support et l'autorité de groupe ou d'institution qui vient renforcer la légitimité des auteurs ${ }^{43}$.

Nous adopterons ici une perspective un peu différente. L'autorité n'est qu'une des dimensions de l'identité mise en scène par l'auctorialisation. Si l'on s'intéresse à cet aspect, nous distinguons la source de l'autorité associée à la position de l'auteur dans le groupe ou l'institution (relations de pouvoir, d'influence, d'expertise) de sa manifestation dans les dispositifs d'information, par l'auctorialisation textuelle ou de contenu d'une part et l'auctorialisation

\footnotetext{
40 Neeman, Elsa, Jérôme Meizoz, et Claire Clivaz. "Culture numérique et auctorialité : réflexions sur un bouleversement ». A contrario, no 17 (17 août 2012): 3-36 et Candel, Étienne, et Pergia Gkouskou-Giannakou. «S'instituer par l'écriture en ligne». Communication \& langages, no 192 (2017): 19-26. https://doi.org/10.4074/S0336150017012029.

${ }^{41}$ Op. Cit. Zacklad 2005.

42 Broudoux, Evelyne. "Autoritativité, support informatique, mémoire ». Journées " hypertextes, mémoire, fiction » Montréal, novembre 2003, 31 octobre 2003. https://archivesic.ccsd.cnrs.fr/sic 00001137/document.

43 Broudoux, Evelyne. "Construction de l'autorité informationnelle sur le web». In A Document (Re)turn: Contributions from a Research Field in Transition, édité par Roswitha Skare, Niels Windfeld Lund, et Andreas Värheim, 12. Peter Lang, 2007.
} 
documentaire d'autre part. L'auctorialisation documentaire inclue elle-même toutes les facettes de la documentarisation, auctoriale, éditoriale, diffusionnelle, d'appropriation (cf. infra).

En contexte numérique et notamment dans les médias sociaux, les enjeux de l'auctorialisation documentaire correspondent à l'identité numérique ${ }^{44}$, la présence numérique ${ }^{45}$, ou la réputation numérique ${ }^{46}$ qui dépendent en partie des calculs algorithmiques basés sur les likes et autres «retweet» comme sur les stratégies de référencement des moteurs. Selon Camille Alloing, la réputation se distingue de l'autorité informationnelle parce qu'elle relève d'une "métaopinion » agrégeant différents éléments de popularité ou de mise en visibilité au sein d'un collectif. Cette approche rejoindrait pour nous la problématique de la documentarisation diffusionnelle (cf. infra), celle du libraire, du bibliothécaire, voire du journaliste qui construit une mise en visibilité pour un public donné à partir de différentes sources éditoriales.

\section{L'information support et la division du travail de documentarisation : auctorial, éditorial, diffusionnel, d'appropriation}

Nous sommes maintenant en mesure de préciser la définition de la documentarisation que nous avons donnée plus haut. Rappelons d'abord que la documentarisation peut être interne ou externe. Dans le premier cas elle est essentiellement orientée vers l'organisation des fragments sémiotiques à l'intérieur d'une unité de production sémiotique cohérente, un livre, un rapport, un blog, dans un dispositif de médiation informationnel pour en faciliter la lecture et l'interprétation. Dans le second, elle est essentiellement orientée vers la mise en relation de plusieurs unités de production sémiotique de manière à les classer les unes par rapport aux autres pour en faciliter l'accès.

Bien sûr, avec le numérique, avec la généralisation des liens hypertextes et l'écriture dans des systèmes de gestion de contenus qui hébergent des forums, des blogs, des wikis et des systèmes de réseaux sociaux, la question de la définition des unités de production cohérentes est une question en soi que nous ne pouvons pas aborder ici. Mais globalement, on peut toujours considérer que certains systèmes d'organisation des connaissances, dit internes, sont d'abord dédiés à

44 Ertzscheid, Olivier. "L'homme, un document comme les autres ». Hermès, no 53, 2009, p. 33. Crossref, doi: $10.4267 / 2042 / 31473$.

45 Merzeau, Louise. «Présence numérique: les médiations de l’identité ». Les Enjeux de l'information et de la communication, 2009, no 1 (2009): 79-91.

46 Alloing, Camille. «La fabrique des réputations selon Google ». Communication \& langages, no 188 (2016): 101-22. https://doi.org/10.4074/S0336150016012060. 
faciliter la lecture tandis que d'autres, dit externes, sont d'abord dédiés à faciliter le classement et la recherche depuis « l'extérieur» du texte, même si d'un point de vue technique, les deux fonctions se rejoignent de plus en plus.

Ainsi, alors que la fonction d'aide à la navigation à l'intérieur du contenu sémiotique sert essentiellement la documentarisation interne, les fonctions d'identification du contexte transactionnel et de mise en relation avec les autres documents servent à la fois la documentarisation interne et externe. Voici de manière non limitative quelques Systèmes d'Organisation des Connaissances qui illustrent ces différentes fonctions :

- aide à la navigation : paragraphes, saut de page, numéros de pages, titres et sous-titres, gras, encadrés, table des matières, hyperliens internes, indexation par des moteurs de recherche internes, modèle de page propre à une collection, etc. ;

- identification du contexte transactionnel : auteurs, dates de publication, lieu de publication, dates de modification, service émetteur, etc. ;

- liens avec d'autres documents: citations, notes de bas de page, bibliographie, hyperliens externes, annotations par des systèmes de classification, indexation matière, indexation automatique des par des moteurs, etc.

La « fluidité » des supports numérique va permettre à peu de frais la généralisation des pratiques de redocumentarisation dans le sens spécifique que nous donnons à ce terme :

"Redocumentariser, c'est documentariser à nouveau un document ou une collection en permettant à un bénéficiaire de réarticuler les contenus sémiotiques selon son interprétation et ses usages à la fois selon la dimension interne (...) ou externe (...). Dans ce contexte, la numérisation offre des opportunités inédites pour la réappropriation des documents et des dossiers en vue de satisfaire les intérêts de nouveaux bénéficiaires. ${ }^{\prime \prime 7}$.

Quel que soit la technologie du support, papier ou numérique, la conception et la production des dispositifs informationnels fait l'objet d'une division du travail sophistiquée. La principale différence introduite par le numérique est que dans certaines étapes, la conception est quasiment concomitante de la production à la fois pour la mise en forme de l'apparence finale du texte et pour la diffusion à grande échelle des contenus. Aujourd'hui, dans de nombreux secteurs d'activité, les auteurs mettent le plus souvent directement en forme le texte qui sera diffusé sans passer par toute une série d'étapes intermédiaires. Mais ils le font souvent

47 Zacklad, Manuel. "Réseaux et communautés d’imaginaire documédiatisées ». A Document (Re)turn, édité par Roswitha Skare et al., Peter Lang, 2007, p. 279-97. HAL Archives Ouvertes, https://archivesic.ccsd.cnrs.fr/sic 00180185. 
sur la base de modèles, de templates, d'architextes ${ }^{48}$, de systèmes d'organisation des connaissances qui ont été conçus par d'autres acteurs.

De même, les systèmes de gestion de contenu ou les réseaux sociaux permettent immédiatement de diffuser à un large public les textes mis en forme sans passer par de longues phases d'impression papier. Le phénomène est encore plus marqué dans les entreprises et les administrations. Le régime des documents pour l'action ${ }^{49}$ exploitant la messagerie, les systèmes de gestion de contenu et les systèmes de rédaction collaborative dans le nuage, permette une co-conception rapide et une diffusion tout aussi immédiate de l'information semi-structurée qui est au cœur des activités tertiaires à haute valeur ajoutée.

Mais, en relation assez directe avec les étapes et les compétences qui étaient mises en avant dans les dispositifs informationnels pré-numériques, une analyse attentive permet de révéler une division du travail sophistiquée dans la conception des dispositifs numériques correspondant à différentes dimensions du design de l'information documentaire. Nous considérons cinq principaux rôles ou métiers au sein du travail de documentarisation qui prolongent la textualisation pour permettre la structuration des contenus et leur diffusion: auteur, éditeur, imprimeur/programmeur, diffuseur, lecteur.

Les trois premiers, auteur, éditeur, diffuseur, correspondent au travail de design de l'information ou d'une méta-information pour le diffuseur. Nous ne traitons pas ici du travail de l'imprimeur/programmeur malgré son importance et par manque de temps. Il se situe dans les phases aval de la conception qui ne sont pas directement en lien avec le design systémique au sens où nous l'avons défini, même si les interactions entre les programmeurs des systèmes de gestion de contenu ou des plateformes et les designers peuvent être très importantes ${ }^{50}$.

Une des différences les plus notable entre les dispositifs numériques et les dispositifs papiers est que les auteurs et les lecteurs peuvent agir sur les supports qui sont à la fois des environnements d'écriture, d'édition et de diffusion et influencer celle-ci de manière plus directe et rapide que dans les agencements collectifs classiques du marché des œuvres papiers où la régulation de l'offre et de

\footnotetext{
48 Jeanneret, Yves, et Emmanuël Souchier. «L'énonciation éditoriale dans les écrits d'écran ». Communication \& Langages, vol. $145, \mathrm{n}^{\circ} 1,2005$, p. 3-15. wmw.persee.fr, doi:10.3406/colan.2005.3351.

49 Zacklad, Manuel. « Documentarisation Processes in Documents for Action (DofA): The Status of Annotations and Associated Cooperation Technologies ». Computer Supported Cooperative Work (CSCW), vol. 15, no 2-3, 2006, p. 205-28. link.springer.com, doi:10.1007/s10606-006-9019-y.

${ }^{50}$ Le paramétrage, la mise en forme d'un texte avec des balises, le choix des couleurs et de la typographie relève des compétences des designers même si la coopération avec les informaticiens peut être nécessaire pour ajuster le rendu final selon les environnements-supports logiciels.
} 
la demande est plus lente. Voici en quelques mots la description de ces différents rôles ${ }^{51}$ :

1. Auteur, documentarisation auctoriale : l'auteur a d'abord en charge l'essentiel du travail de textualisation, qu'il relève de l'écrit ou d'autres formes d'expression. Mais il intervient également dans la mise en forme et la structuration du texte sur le support c'est-à-dire dans la documentarisation. Cette prérogative est aussi celle de l'éditeur qui définit souvent des modèles d'architexte, les feuilles de style, les champs des formulaires. L'auteur est néanmoins au cœur de la définition de l'organisation des connaissances adaptés au texte qu'il produit, par exemple le plan, et son écriture est directement impactée par les outils d'annotation, notamment typographiques, qui lui permettent d'organiser sa pensée: marques de paragraphes, de titre et de sous-titre, renvois, etc ${ }^{52}$ La rencontre potentiellement conflictuelle entre l'organisation des connaissances portée par l'auteur et celle portée par l'éditeur est au cœur de la question de l'éditing que nous examinerons plus bas.

2. Editeur, documentarisation éditoriale ou éditorialisation : l'éditeur a en charge la structuration d'une collection de textes pour les inscrire dans des genres cohérents. Ce travail est essentiel pour permettre la rencontre avec les bénéficiaires et faciliter l'appropriation. On peut penser aux nombreux genres des manuels scolaires ou universitaire, des guides de voyage, des livres de cuisine, des manuels d'utilisation, des dictionnaires, des articles scientifiques, etc. Le passage au numérique implique une transposition de ces genres tenant compte des nouveaux moyens offerts par cette technologie et de la créativité des développeurs de systèmes de gestion de contenu. La difficulté de la situation actuelle est que les nouveaux genres propres à l'écrilecture numérique ${ }^{53}$ ne sont pas stabilisés même si on peut repérer de nouveaux genres éditoriaux comme dans la pratique des $\operatorname{blogs}^{54}$. Dans la terminologie fluctuante des métiers du web, le responsable du contenu, qui correspondrait à l'auteur, est souvent proche du responsable éditorial et il arrive qu'il cumule des responsabilités d'écriture et de définition de la ligne éditoriale. Mais plus les entreprises sont importantes plus les fonctions tendent à se spécialiser.

\footnotetext{
${ }^{51}$ Nous proposons un tableau actualisant en partie ces rôles en contexte numérique dans : Manuel Zacklad «Participative documentary spaces and governance ». International Journal of Sustainable Development, vol. 11, n 2-4, janvier 2008, p. 247-61 ou Espace documentaire participatif et gouvernance. 2007. hal.archives-ouvertes.fr, https://archivesic.ccsd.cnrs.fr/sic_00202423/document.

${ }^{52}$ Kembellec, Gérald, et Bottini, Thomas. «Réflexions sur le fragment dans les pratiques scientifiques en ligne : entre matérialité documentaire et péricope ». In $20^{\circ}$ Colloque International sur le Document Numérique: CiDE.20. Villeurbanne, France, 2017. https://hal.archives-ouvertes.fr/hal-01700064.

53 Kembellec, Gérald, et Évelyne Broudoux, éditeurs. Écrilecture augmentée dans les communautés scientifiques: humanités numériques et construction des savoirs. ISTE editions, 2017.

${ }^{54}$ Cardon, Dominique, et Hélène Delaunay-Téterel. «La production de soi comme technique relationnelle ». Réseaux, vol. no 138, nº 4, décembre 2006, p. 15-71. Caim.info, doi:10.3917/res.138.0015.
} 
3. Libraire, bibliothécaire, référenceur, documentarisation diffusionnelle : le libraire, le bibliothécaire, le marchand de journaux ont pour rôle de distribuer mais aussi de permettre l'accès à un nombre très important de documents à destination de de publics très divers en assurant une médiation diffusionnelle ${ }^{55}$. Cette mise à disposition est à l'intersection d'une logique épistémique et d'une logique commerciale, notamment dans le cas du libraire qui est de plus en plus souvent un libraire en ligne. La documentarisation externe, en particulier l'explicitation du contexte dans lequel le document a été produit et la recherche de systèmes d'organisation des connaissances en mesure d'orienter les lecteurs est au cœur de ces rôles et de leur compétence. C'est un domaine dans lequel les standards de métadonnées de la bibliothéconomie (p.e UNIMARC) jouent un rôle essentiel. En environnement numérique elle rejoint les initiatives du web sémantique et des formats de données liées. Elle correspond également aux nouveaux métiers du référencement et de l'animation de communauté.

4. Lecteur, documentarisation d'appropriation: en environnement papier, les modalités d'appropriation du support par les lecteurs, les annotations qu'ils laissaient sur le livre, relevaient d'une pratique privée sauf dans le cas des bibliothèques où le même ouvrage lu et annoté par un lecteur était emprunté par un autre lecteur. En environnement numérique, le propre des dispositifs infocommunicationnels est d'afficher directement les commentaires des lecteurs dans les plateformes de blog ou de réseaux sociaux numériques. Dans ces plateformes, le suivi de son « audience » est une préoccupation majeure des auteurs. Ce phénomène est encore renforcé dans les plateformes de réseaux sociaux ou les « likes » sont utilisé par les algorithmes de modération pour pousser les articles auprès d'autres internautes. En retour, ces annotations peuvent être directement utilisées par les auteurs, les éditeurs et les diffuseurs.

Une des spécificités du régime numérique est que l'auteur peut facilement assumer l'éditorialisation et la documentarisation diffusionnelle ce qui pourrait laisser à penser que ces distinctions ne sont plus pertinentes. Mais si certains amateurs, chercheurs, journalistes, blogueurs, parviennent à assumer avec talent l'ensemble de ces fonctions, il n'en est pas de même dans de nombreux milieux professionnels où elles correspondent souvent à des métiers assez clairement différenciés. Par ailleurs, quelques soient les contextes, dès que l'on quitte les environnements extrêmement formatés des grands réseaux socio-numériques, il existe le plus souvent une différence entre les éditeurs concepteurs de modèles, de formats, d'architextes et les auteurs, comme il en existe entre les

\footnotetext{
55 Payeur, Cécile, et Manuel Zacklad. «Dispositifs d'articulation entre espaces physique et virtuel pour accéder à l'offre de presse ». Études de communication. langages, information, médiations, no 30, octobre 2007, p. 39-53. journals.openedition.org.proxybib.cnam.fr, doi:10.4000/edc.446.
} 
administrateurs en charge de définir les droits d'accès et d'écriture et les éditeurs et auteurs.

\section{Conclusion : les conflits d'éditing, confrontation entre l'autorité auctoriale et éditoriale}

Dans ces contextes professionnels nombreux où subsiste une division du travail entre les acteurs chargés de la textualisation et ceux chargés de la documentarisation éditoriale et diffusionnelle, voire entre le même acteur pouvant être amené à changer de rôle au fur et à mesure de la progression de son projet, la gestion des interactions entre les processus de textualisation et de documentarisation correspond pour nous à la question de l'éditing, un terme que nous reprenons directement de l'anglais. Selon les médias, l'éditing ou révision, montage, normalisation, mastering, correspond à l'adaptation du texte à la structure portée par le support, structure qui, comme nous l'avons vu, correspond à des choix d'organisation des connaissances plus ou moins explicites. Cette adaptation peut prendre des formes très diverses selon le degré de standardisation de la transaction et des dispositifs qui la médiatise. Dans le domaine de l'écrit ou pourra penser aux différents formats que sont les questionnaire, formulaire, plan type, modèles de styles, format, templates, gabarits et autres structures documentaires prédéfinies.

Les conflits potentiels entre textualisation et documentarisation sont le plus souvent liés à la documentarisation éditoriale dite aussi éditorialisation. On sait que ce terme a connu un regain d'intérêt corollaire de la multiplicité de ses significations allant de «l'énonciation éditoriale» promue par E. Souchier ${ }^{56}$ jusqu'à la signification très englobante promue par Vitali-Rosati ${ }^{57}$ à la suite de Bachimont ${ }^{58}$, qui correspond en fait à notre définition de la redocumentarisation rappelée plus haut ${ }^{59}$, en passant par celle de B. Guyot ${ }^{60}$ et bien sur notre propre acception de la «documentarisation éditoriale $»^{61}$. Bien que E. Souchier ${ }^{62}$ ne fasse

\footnotetext{
${ }^{56}$ Souchier, Emmanuël. «L'image du texte pour une théorie de l'énonciation éditoriale ». Les cahiers de médiologie, $\mathrm{n}^{\circ}$ 6, 1998, p. 137-45. Cairn.info, doi:10.3917/cdm.006.0137.

57 Vitali Rosati, Marcello. «Qu'est-ce que l'éditorialisation?» Sens Public, mars 2016. sens-public.org, http://senspublic.org/article1184.html.

58 Bachimont, Bruno. «Nouvelles tendances applicatives: de l'indexation à l'éditorialisation ». L'indexation multimédia: description et recherche automatiques, édité par Patrick Gros, Hermès science publications-Lavoisier, 2007. ${ }^{59}$ Ibid Zacklad 2007a.

${ }^{60}$ Guyot, Brigitte. «Processus éditorial : faire passer un document d'un monde à l'autre ». Où va le travail à l'ère du numérique?, édité par Anne-France de Saint-Laurent Kogan et Jean-Luc Metzger, Mines Paris, les presses-Paristech, 2007.

61 Zacklad, Manuel. Espace documentaire participatif et gouvernance. 2007. hal.archives-ouvertes.fr, https://archivesic.ccsd.cnrs.fr/sic 00202423/document.

${ }^{62}$ Souchier, Emmanuël. «L'image du texte pour une théorie de l'énonciation éditoriale ». Les cahiers de médiologie, $\mathrm{n}^{\circ}$ 6, 1998, p. 137-45. Cairn.info, doi:10.3917/cdm.006.0137.
} 
pas référence à la notion de document pour rendre compte de la fonction des supports, c'est bien de sa définition dont nous pourrions être le plus proche, résumée ici par Pascal Genêt ${ }^{63}$ :

"D'un point de vue sémiologique, l'énonciation éditoriale repose sur deux caractéristiques: la pluralité des instances intervenant dans la constitution et la médiation d'un texte (auteur, éditeur, média, etc.) et le processus de dissimilation des marques d'énonciation éditoriale à travers ce que Soucbier qualifie l'" image du texte », c'est-à-dire "une interdétermination du sens et de la forme [...] qui participe activement de l'élaboration des textes » (Souchier, 1998, p. 138). L'énonciation éditoriale forme ainsi un "texte second» dans la mesure où "le signifiant n'est pas constitué par les mots de la langue ("texte premier"), mais par la matérialité du support et de l'écriture, l'organisation du texte, sa mise en forme " (Souchier, 1998). Ce concept permet d'apprébender "l'image du texte » comme le résultat d'un processus d'ensemble: des intentions lors de la création et de la mise en forme (rôle de l'editor dans l'écriture) à l'influence de l'empreinte de la matérialité des supports sur les usages et pratiques (le rôle du publisher dans la lecture)."

Cependant, ce que manque peut-être en partie E. Souchier qui s'attache essentiellement à la forme du texte, la dimension typographique de la documentarisation, c'est le fait que l'éditorialisation, comme les autres formes de documentarisation, ne relève pas exclusivement d'un processus de dissimulation par lequel auteur, éditeur et diffuseur, à travers les formats des architextes, influencent le sens du texte d'une manière difficilement identifiable par le lecteur mais également par eux-mêmes, en étant les jouets de choix effectués en amont par les éditeurs de logiciels. Sans remettre en cause le bien-fondé de cette vision, il faut parallèlement envisager le fait que l'éditorialisation et les autres formes de documentarisation peuvent relever, au contraire, de processus délibérés, explicites et revendiqués, visant à assurer le transfert d'une autorité de contenu ou d'une autorité documentaire en exploitant les codes d'un genre au service de ses intérêts.

Par ailleurs, l'éditorialisation n'est pas que typographique. Elle correspond à la production d'un paratexte spécifique dédié à la gestion logistique et intellectuelle du support, dont la forme typographique ne représente qu'une dimension. Dans les environnements numériques, ce paratexte documentaire qui englobe une très grande variété de métadonnées relevant de systèmes d'organisation des connaissances plus ou moins standardisés, contribue à la forme du texte mais également à son référencement, sa «trouvabilité », sa «navigabilité », sa visibilité dans les réseaux. Comme il est souvent généré en partie automatiquement, ce qui atteste du caractère en partie dissimulé que souligne E. Souchier, il tend à être

63 Genêt, P. (s. d.). Énonciation éditoriale. Consulté 3 octobre 2018, à l'adresse http://ressourcessocius.info/index.php/lexique/21-lexique/190-enonciation-editoriale 
aussi important en volume que le texte brut lui-même qui peut être ininterprétable s'il n'est pas documentarisé.

Comme nous l'avons déjà souligné, parmi les différents acteurs qui contribuent à la documentarisation, l'éditeur a pour fonction de gérer la cohérence des collections d'unités de production sémiotique, en définissant des genres de référence qui faciliteront l'appropriation par le lecteur, mais également la textualisation, le travail de l'auteur. Son autorité s'appuie principalement sur l'auctorialisation documentaire et est issue de son rôle au sein d'agencements collectifs propres à des secteurs ou à des domaines d'activité. On pourra ainsi, par exemple, au sein du travail d'éditorialisation, faire prévaloir une dimension territoriale (source d'autorité étatique ou juridique), marchande ou ouverte sur un public au sens large (source autorité artistique, journalistique, ou portée par une marque commerciale), organisationnelle (source d'autorité managériale), communautaire (source d'autorité liée à la tradition ou à un objectif militant), etc.

Quels que soient les dispositifs, informationnel, infocommunicationnel, ou transmédia, le responsable de l'éditorialisation construit l'architecture de l'information qui peut être élargie, dans le cas d'un dispositif transmédia, à l'architecture spatiale et temporelle d'un évènement ou d'un processus comme à celles des séquences communicationnelles en présentiel qu'il inclut. L'éditeur, dans la perspective élargie de la documentarisation éditoriale, promeut une organisation des connaissances qu'il instancie dans différents supports pour contraindre, mais aussi habiliter, le travail de textualisation auctoriale. Notons que, dans notre perspective, l'auteur peut être également porteur d'un projet de documentarisation, c'est-à-dire qu'il peut revendiquer une forme de structuration personnelle du support qui peut être plus ou moins libre selon la rigidité de la structure et les droits d'accès qui lui sont conférés. On retrouve la complémentarité décrite par Clot et Faïta ${ }^{64}$ entre genre et style appliquée aux genres professionnels « Chaque sujet interpose entre lui et le genre collectif qu'il mobilise ses propres retouches du genre. Le style peut donc être défini comme une métamorphose du genre en cours d'action. $»^{65}$.

${ }^{64}$ CLOT, Yves, et Daniel FAÏTA. « Genres et styles en analyse du travail Concepts et méthodes ». Travailler, vol. 4, 2000 , p. 36.

${ }^{65}$ Ibid. 